\title{
Teoría del Apego y Síndrome de Down: conceptos básicos y claves educativas
}

\author{
Yanina Guerrero y Eduardo Barca-Enríquez \\ Universidade da Coruña
}

\begin{abstract}
Resumen
El nacimiento de un hijo generalmente predispone a los padres para desarrollar una crianza adecuada. Sin embargo, cuando el bebé expresa una alteración en su desarrollo que "dista de la evolución esperada", se rompen las expectativas habituales y el proceso de vinculación afectiva puede verse mermado. Se altera así el desarrollo de un apego seguro. Pretendemos recopilar y organizar toda la información bibliográfica al respecto, resaltando las principales claves para favorecer un apego seguro en niños con Síndrome de Down, permitiendo un mejor desarrollo integral con el objetivo de posibilitar el logro de los mayores potenciales de cada uno.

Palabras clave: Síndrome de Down, teoría del apego, situación del extraño, claves educativas
\end{abstract}

En lo que se refiere al ámbito de la intervención de corte psicosocial y clínica, es más que corriente recurrir a la Teoría del Apego para basar la explicación de la relación entre un menor y sus cuidadores. Dicha teoría tiene sus raíces en el psicoanálisis, en la etiología y en las teorías cognitivas-conductuales, y fue organizada y sistematizada por Bowlby. Este considera que el niño viene al mundo preparado biológicamente para establecer un estrecho lazo de unión con su figura de cuidado. Este autor formula los supuestos básicos de su teoría sobre la motivación y la regulación de la conducta. Considera que existen diversos sistemas conductuales que están diseñados para alcanzar una meta concreta. De esta forma, la función del sistema conductual del apego es la supervivencia del individuo y de la especie en general.

Las aportaciones de Mary Main y colaboradores, junto con los estudios de Ainsworth, ayudan a la expansión de dicha teoría añadiendo un estilo más y dando así mayor consolidación a lo que parecía ser una interesante y enriquecedora línea de trabajo. Ainsworth (1983), define el apego como aquellas conductas que favorecen ante todo la cercanía con una persona determinada. Es mutuo y recíproco. Diseña una de las técnicas más fructíferas en el estudio del apego como es la Situación Extraña, la cual contribuye a que Mary Main descubra una nueva categoría de estilos de apego. Así Bowlby, Ainsworth y Main serán las figuras con mayor peso en el tema del apego, y quienes mayores contribuciones han hecho a este ámbito.

\section{El apego en niños con Síndrome de Down}

El nacimiento de un hijo suele ser un acontecimiento que genera satisfacción y alegría. Sin embargo, cuando el bebé expresa una diferencia visible y notoria que "se aleja de la normalidad", se rompen las expectativas y el proceso de vinculación afectiva puede verse modificado o mermado. Esta situación se hace más pronunciada cuanto más visibles sean las discapacidades que presente el bebé, como ocurre en los casos de Síndrome de Down.

Para evitar casos de maltrato, abandono o discriminación, concretamente en el caso de niños con Síndrome de Down, es necesario que el equipo de salud perinatal elabore anticipadamente estrategias que faciliten y refuercen en el momento en el que se diagnostica al pequeño, el apego y la vinculación afectiva, principalmente después del parto. En caso de niños autistas, deberá tenerse en cuenta todas estas directrices en el momento de su diagnóstico y comunicación a sus progenitores. Se considera fundamental y primordial cuidar el lenguaje que se emplea, la información que se brinda y respetar las etapas emocionales por las cuales atraviesan los padres. Es importante atender estos aspectos puesto que la actitud de sus padres influirá con casi absoluta seguridad en su capacidad de integración social y emocional del hijo a la familia y a la sociedad.

Como sabemos, el proceso de vinculación afectiva es algo conocido y su origen es ancestral. Se ha determinado además que esta conducta es predecible y evolutiva, esto es, que se produce por etapas secuenciadas (Rossel, 2004).

\section{Etapas de la vinculación afectiva}

- Primera etapa: corresponde al periodo del embarazo. Durante la gestación y los momentos previos al parto los padres se encuentran ansiosos y cargado de expectativas positivas con respecto a su hijo. Ese cariño y afecto que se ha ido formando a lo largo de 9 meses tomará una forma tangible con el nacimiento del bebé. Se idealiza al bebé, se piensa y se divaga sobre diversos aspectos de la vida del futuro hijo. Se dice que se produce un "enamoramiento" de la imagen del bebé ideal.

- Segunda etapa: comienza inmediatamente después del parto. En esos momentos, los padres, quienes están cargado de expectativas positivas sobre su bebé, toman contacto real y directo con el mismo por primera vez. El recién nacido, al ser puesto sobre el abdomen de su madre, posee la capacidad innata de dirigirse hacia el pezón y succionar firmemente. Este evento que se produce dentro de la primera media hora de vida, es extremadamente importante para solidificar la vinculación afectiva, puesto que es cuando se producen crecientes descargas de oxitocina a nivel cerebral, se elevan además lo niveles plasmáticos de opiodes endógenos, se desencadena un estado de somnolencia y 
ansiedad en la madre con un elevado umbral para el dolor. Se trata así de un estado emocional difícil de describir donde la madre, al ver y sentir a su hijo, completa y consolida la fase de enamoramiento que se inició en la fase de embarazo, y que marcará la maternidad de forma positiva

- Tercera etapa: se inicia después del nacimiento. Es el periodo más largo y evolutivo de los tres, y en el cual se refuerzan los lazos afectivos que se establecieron en un primer momento a través del proceso del apego. Como sabemos, este proceso se caracteriza por la interacción de ambas partes para que el resultado sea adecuado y óptimo. Cabe destacar, como se ha comentado anteriormente, que el niño se va desarrollando y va creciendo, a la vez que recibe los estímulos de sus padres y responde de acuerdo a su etapa de desarrollo.

\section{Factores que alteran el proceso de vincultación y el desarrollo normal del vínculo de apego}

- Los padres: condiciones de salud de los progenitores desfavorables, principalmente de la madre, que imposibiliten el contacto inicial. Ejemplo de ello sería casos de infecciones, cesárea, hipertensión arterial severa, entre otros. También incluiríamos en este apartado la salud mental y la estabilidad emocional de la madre, por ejemplo, deterioro neurológico, madre adolescente, drogadicción...

- Medio ambiente: hospital con normas rígidas que entorpecen el contacto precoz de los bebés con sus padres (visitas restringidas, falta de contacto directo con el bebé, entre otros); equipo médico poco acogedor y mal informado (desconoce las redes de apoyo social y los programas de estimulación neuro-sensorial locales,...); ausencia de redes de ayuda social de accesibilidad local (capacidad de integración social,...); medio social y cultural intelectualmente desprotegido (véase drogadicción, alcoholismo, prejuicios,...) junto con las experiencias previas desfavorables.

- Recién nacido: condiciones de salud del bebé que imposibilitan la permanencia con su madre. Estas condiciones pueden ser transitorias, prolongadas $\mathrm{o}$ permanentes.

\section{Etapas de transición emocional de los padres con hijos con discapacidad}

Se diferencian diversas etapas emocionales por las cuales atraviesan los padres cuando tienen un hijo con discapacidad (Rossel, 2004):

- Primera etapa, impacto: la noticia del diagnóstico de su bebé los deja paralizados, no logrando comprender qué está pasando. Sienten que sus expectativas positivas sobre su hijo se han esfumado y parecen vivir una pesadilla que dista de la realidad. Se encuentran atónitos, perplejos. Es posible que rompan a llorar. Unos optan por preguntar insistentemente por la condición de su hijo, mientras que otros hacen como si ésta no existiese. Existen dificultades y están reacios a acoger a su bebé, principalmente si no han tenido contacto inmediato posterior al parto puesto que la imagen de su bebé es "desfavorable" a lo que se esperaban.
Cuanto mayor sea el periodo de distanciamiento posterior al parto, mayores serán dificultades tendrán para enfrentar a su hijo

- Segunda etapa, negación: posteriormente, los padres atraviesan una etapa de negación de la realidad. Se resisten a aceptar la situación de su hijo. Es posible que hasta lleguen a verbalizar "que éste no es su hijo" o "debe de ser un error", "no tiene lo que dicen", "están equivocados", entre otros. Los prejuicios y las opiniones de terceros cobran importancia. Existen casos donde los padres permanecen incomunicados y separados, de forma que se dificultará aún más la vinculación.

- Tercera etapa, tristeza o dolor: los padres ya toman conciencia de la realidad e intentan comprender en parte lo que les ocurre. Se va consolidando paulatinamente la imagen de pérdida del bebé ideal, de los sueños esfumados y perdidos y de la esperanza ya inexistente. Todo ello conllevará un profundo sentimiento de dolor, tristeza y angustia. Pueden expresarlo mediante impotencia, frustración y posiblemente sentimientos de culpabilidad. Se encuentran realmente angustiados, le temen a lo desconocido.. Sufren cuando perciben la dificultad de su vinculación, aunque son más próximas que en las etapas anteriores y acceden a tomar a sus hijos, amamantarlos y expresan sentimientos de ternura y dolor a la vez.

La duración de esta etapa es variable y depende en gran parte de cómo han sido resueltas las etapas anteriores. Los padres buscarán en sus hijos señales de interacción (succión energética al pecho, una sonrisa, fijación de la mirada,...). Sin embargo, es posible que no todos los bebés que padecen Síndrome de Down pueden responder a estas demandas al nacer debido a su hipotonía. Ello conlleva frustración de los padres y los desalienta enormemente, llegando incluso a creer que su hijo "no los necesita". Esta ansiedad, junto al dolor que experimentan, propicia a que ciertos padres pierdan las esperanzas de vincularse con su bebé.

- Cuarta etapa, adaptación: entran en un proceso de adaptación que se caracteriza por aceptar la condición de su hijo y los sentimientos que están experimentando. Se muestran interesados en aprender y conocer más sobre el Síndrome de Down. Es de suma importancia la aceptación que el medio familiar ha expresado a este nuevo integrante así como el apoyo que la pareja exprese al otro miembro. El peso del ambiente, en caso de ser nocivo y negativo, puede exponer al niño a riesgos de desvinculación afectiva, abandono o maltrato.

- Quinta etapa, reorganización: el bebé ha sido completamente integrado a la familia, la cual ha aprendido a conocer y tolerar sus discapacidades y se comprometen e implican en su rehabilitación y en su adaptación al medio. La vinculación afectiva se construye a la par que se fortalece cada día mediante los avances emocionales y neurológicos del bebé, facilitando así la interacción con sus padres, convirtiendo así este proceso en una retroalimentación positiva. Los programas de estimulación temprana facilitarán la consecución de esta retroalimentación, en la cual los padres tienen la oportunidad de relacionarse con un 
equipo multidiscilplinar y con otros padres en situaciones similares a la suya.

Cabe destacar que la temporalidad con la que se producen las anteriores etapas no está muy bien definida. La duración de cada una de ellas varía y dependerá principalmente de la influencia del medio ambiente sobre los progenitores y los rasgos emocionales de los mismos. Es posible que los padres permanezcan más tiempo o incluso se queden estancados emocionalmente en alguna de las etapas iniciales (en las tres primeras), impidiéndoles avanzar hacia las etapas de resolución y adaptación.

\section{Investigación sobre Apego y Síndrome de Down}

A su vez, diversos autores han abordado y estudiado el papel del apego en niños con Síndrome de Down. En concreto, Beeghly, Weiss-Perry, \& Cicchetti (1990) consideraban que la construcción por parte de estos niños de unas relaciones seguras de apego entre los doce y los veinticuatro meses era la consecuencia de la interacción con sus cuidadores, siendo así el apego seguro el patrón normativo de los niños con esta discapacidad. Sin embargo, se crean ciertas dudas sobre la validez de la Situación Extraña como medio de evaluación del apego en estos niños puesto que muchos de ellos no parecen denotar estrés durante los episodios de separación y reunión (Vaughn y Waters, 1990), aunque los pequeños son conscientes de la separación.

Otros profesionales como Main y Solomon (1990) indicaron que no es conveniente utilizar la categoría de apego desorganizado cuando se investigan a niños con discapacidades físicas y/o mentales ya que muchos de ellos pueden presentar elementos de desorganización conductual. Esta desorganización puede ser resultado más de su discapacidad que del fracaso en la construcción de una estrategia coherente de organización de la conducta de apego.

Vaughn, Goldberg, Atkinson, Marcovitch, MacGregor y Seifer (1994) estudiaron tres muestras independientes con Síndrome de Down de entre doce y veinticuatro meses de edad. Se empleó para su evaluación la Situación Extraña. Los resultados que se obtuvieron fueron los siguientes: el $42 \%$ de los niños no pudo ser clasificado en un tipo de apego concreto utilizando el procedimiento tradicional, siendo categorizados como inseguros/inclasificables. Además, se muestran diferencias significativas entre ambos grupos en seguridad del apego. Así, era más probable que los pertenecientes al grupo de comparación presentaran un apego seguro, aunque la tasa superior de inseguros entre los niños con Síndrome de Down podría atribuirse a la imposibilidad de clasificar a muchos de ellos. Incluso cuando se descartan estos casos inclasificables de los análisis, el porcentaje en el grupo de comparación fue mayor que entre los del Síndrome de Down (38\% frente a un $21 \%$ ). Se encuentran diferencias también en la distribución de los tres tipos de apego. Esto puede deberse fundamentalmente a la baja proporción de ambivalentes entre los niños con este síndrome. Se observan diferencias en las escalas de interacción, esto es, en la búsqueda de proximidad, mantenimiento del contacto, resistencia al contacto/interacción y en el número de intervalo de llanto. Los niños que padecían Síndrome de Down puntuaron menos en estas escalas, excepto en la conducta de evitación.

Como se ve, son unos resultados poco esclarecedores y su interpretación resulta algo compleja. Por un lado, podemos pensar que las diferencias observadas son superficiales y que con una selección adecuada de sujetos se encontrarían unos patrones de apego similares. Los niños con Síndrome de Down se apegan a sus madres y la mayoría de ellos establece unas relaciones seguras de apego con sus progenitoras.

Vaughn et al. (1994) interpretaron los resultados de un modo totalmente diferente al que hemos explicado líneas arriba. Ellos consideran que la Situación Extraña provoca en los niños con este síndrome unos niveles de estrés funcionalmente distintos. Defienden que ellos raramente exteriorizaban su estrés (ni siquiera cuando se quedaban solos). Este nivel bajo de activación/estrés propiciaba que no buscaran el contacto durante los reencuentros, aunque se aproximaran e interactuaran. Además, la falta de contacto y de necesidad de que los tranquilizaran hacían que difícilmente presentaran una conducta de resistencia.

Así, se considera que el diferente impacto psicológico de los episodios de separación y reunión pone de manifiesto la incapacidad del procedimiento de Ainsworth (seguro, evitativo, preocupado) y de Main y Solomon (desorganizado) para la captación de las capacidades y diferencias y no evalúen el patrón de apego de estos niños. De esta forma, este procedimiento no debería utilizarse ni para evaluar ni para valorar de forma rutinaria la conducta en los niños con Síndrome de Down en la Situación Extraña.

También puede ocurrir que los procesos necesarios para la consolidación del apego requieran más tiempo en el caso de estos niños. Debemos destacar además dos estudios de niños con Síndrome de Down en los que la calidad de las relaciones de apego se evaluó con el Attachment Q-set (AQS; Waters y Deane, 1985) y con la Situación Extraña. Vaughn, Leftover, Seifer \& Barglow (1989) demuestran que los tipos de apego según la Situación Extraña no se relacionaban con las puntuaciones en seguridad del apego con las obtenidas en el AQS. Esto podría ser una prueba de que las clasificaciones definidas en la Situación Extraña no constituyen un índice válido de la seguridad del apego en estos niños.

Sin embargo, en otro estudio, Atkinson y sus colaboradores encontraron una relación significativa entre el tipo de apego obtenido en la Situación Extraña y la puntuación obtenida en seguridad en el AQS cuando los niños tenían veintidós meses de edad. Por lo tanto, cabe la posibilidad que los procesos de consolidación del apego en niños con Síndrome de Down lleven más tiempo. Atkinson et al. (1999) evaluaron la influencia del funcionamiento adaptativo/intelectual del niño y de la sensibilidad de la madre, y la interacción entre ambas variables, sobre la seguridad del apego.

El estudio consistía en medir cuatro constructos en dos ocasiones diferentes durante dos años. Estos cuatro constructos serían los siguientes: funcionamiento cognitivo del niño, seguridad del apego, sensibilidad 
materna y funcionamiento adaptativo. La muestra contó con 53 niños de entre catorce y treinta meses de edad cronológica en la primera observación y sus madres. Siete meses después, al pasar por la situación extraña por primera vez, los niños tenían una edad mental de entre doce y veintitrés meses según el Bayley. La conducta de la Situación Extraña se codificó en los tipos de apego A, B, C, D y U (aquellos que no pudieron ser clasificados en ninguna categoría). Estos últimos se caracterizaban por una aparente despreocupación aun dándose cuenta de que su madre abandonaba la habitación, o incluso si la separación les inquietaba, se tranquilizaban con la presencia de un extraño.

También se observaron las relaciones de la madre y el hijo en el hogar en dos ocasiones cubriéndose el AQS. La sensibilidad de la madre se evaluó en seis ocasiones durante dos años, en diferentes lugares (laboratorio y hogar), diferentes situaciones (mientras contestaba a los cuestionarios, mientras lo alimentaba, mientras se entrevistaba a la madre) y con diversos procedimientos. Se valoran cuatro constructos generales basados en las escalas de Ainsworth: accesibilidad (disponibilidad psicológicamente cuando la necesita el hijo), sensibilidad (respuesta apropiada y rápida), cooperación (intervención mínimamente intrusiva) y aceptación (la madre valora la voluntad del niño). A mayores se utilizó el Maternal Behavior Q-set para evaluar la respuesta de las madres a las señales del hijo cuando tenía cuarenta y dos meses.

Los resultados de este estudio fueron los siguientes: a los veintitrés meses un $40 \%$ mostraba un apego seguro, $47 \%$ no se puedo clasificar en ninguna categoría y un $13 \%$ mostraba claros indicio de apego inseguro. Dieciséis meses después se obtuvo una distribución similar (47,5\%, 32,5\% y 20\% respectivamente). El $62 \%$ fue clasificado en el mismo tipo de apego según la Situación Extraña a los veintiséis y a los cuarenta y dos meses según el AQS. Además, la mayoría no había desarrollado una conducta de base segura incluso en preescolar. Las diversas medidas de sensibilidad materna se relacionaban con los tipos de apego en la Situación Extraña y con la seguridad del apego (AQS) evaluada a los cuarenta y dos meses de edad. Las madres de los niños con apego seguro eran más sensibles que las de los inseguros y que las de los inclasificables.

Por otro lado, el funcionamiento mental predecía la conducta de los niños en la Situación Extraña y la seguridad del apego según el AQS. De esta forma, los que fueran más competentes cognitivamente era más probable que formaran un apego seguro con sus madres. Los análisis de regresión demostraron que la interacción entre sensibilidad de la madre y el funcionamiento cognitivo del niño predecía la seguridad del apego. Así, la obtención de puntuaciones elevadas en las dos variables incrementaba la probabilidad de que se hubiera desarrollado apego seguro. Además, se constata que la conducta de apego mostrada en el laboratorio era un reflejo bastante exacto de la conducta de base segura mostrada en el hogar.

Podríamos concluir pues, en cuanto a los estudios y la determinación de los estilos de apego en niños con
Síndrome de Down, el planteamiento de ciertas dudas sobre la validez de la Situación Extraña en esta población, puesto que muchos de estos niños no parecen experimentar estrés durante estos episodios de separación aunque sean conscientes de ello. Además, la categoría de apego desorganizado no podría utilizarse en estos niños por los motivos ya explicados anteriormente, que se referían que dicha desorganización podría deberse más a la discapacidad que a la falta de una estrategia coherente de organización de la conducta de apego. Se ha constatado que hasta un $42 \%$ de niños con Síndrome de Down no pueden ser clasificados y que puntúan meno en la búsqueda de proximidad, resistencia al contacto/interacción y al mantenimiento del contacto.

Es posible que ocurra también que los procesos necesarios para que se consolide el apego necesiten de más tiempo en caso de niños con esta discapacidad. Atkinson et al. (1999) encontraron que la mayoría no había desarrollado una conducta de base segura incluso en preescolar, ni en caso de tratarse de una circunstancia rutinaria en las que el apego y la exploración estaban libres de estrés (AQS) ni en el caso de dar respuesta a una emergencia (Situación Extraña). La conducta de apego que se muestra en el laboratorio era un reflejo bastante fidedigno de la conducta de base segura mostrada en el hogar del niño. El 47\% no pudo ser clasificado a los dos años, mientras que un $32 \%$ no puedo ser clasificado tampoco a los cuatro años. Se constata también que la interacción entre sensibilidad materna y el funcionamiento cognitivo del hijo predecía la seguridad del apego.

\section{Claves educativas generales}

Con todos los conocimientos y demostraciones de evidencias claras hasta aquí señaladas, consideramos pues que el apego también es una emergencia afectiva en los niños con Síndrome de Down. Por ello, debemos tener en consideración la importancia de un cambio en los modelos afectivos de los padres de dichos niños. Así, es conveniente no sólo comprender cada una de las etapas por las cuales atraviesan los progenitores descritas anteriormente, sino también tenerlas en consideración y respetarlas. No debemos interferir con el curso natural de los eventos de la vinculación, sino que tenemos que hacer todo lo posible por facilitar el contacto físico inmediato posterior al parto entre los padres y los bebés, principalmente con la madre. Simplemente con colocarlo sobre el pecho de la misma, ya estamos iniciando el apego. Todo ello es conveniente de hacerlo antes de brindarle y comunicarles toda la información al respecto de su hijo. Es preferible hacerlo una vez hayan tomado contacto físico con su bebé. Debemos hacer hincapié en que se concentren en las necesidades afectivas, en la alimentación de su bebé, en el calor que le deben brindar a éste, y que la información al respecto de la discapacidad de su hijo se le brindará más adelante.

Es importante señalar que si por diversas razones el bebé debe permanecer separado de sus padres, es recomendable intentar reducir al máximo este tiempo de distanciamiento. Diversos autores señalan que más de 24 horas separados dificultaría el restablecimiento del apego 
puesto que los padres se encuentran en la etapa de impacto. Es conveniente no presionarlos y limitarse a preguntar si desean ver y conocer a su bebé, darle el pecho o cogerlo en brazos. $\mathrm{Si}$ estos responden negativamente a nuestra cuestión, no hay que forzarlos para que lo hagan, ya que estarían en la etapa de negación.

Cuando esta etapa finalice, los padres accederán a coger a su bebé, con miedo pero sin rechazo. Sabemos que éstos necesitan tiempo para superar este duro momento. Si se encuentran en la etapa de negación o de dolor no es conveniente darles demasiados detalles médicos, sino que es más beneficioso brindarles apoyo y preguntarles cómo se siente, propiciando así la expresión de sus emocionales, sentimientos, temores, prejuicios o creencias acerca de la situación por la que pasan, llegando incluso a llorar. En ese momento no debemos ni educarlos, ni hablarles de la condición de salud de su bebé ni parecido, sino arroparlos, acogerlos, familiarizarlos con los sentimientos, con la expresión de los mismos, apoyarlos y animarlos en esta dura circunstancia. Deben encontrarse consigo mismos, enfrentarse a su dolor, ser conscientes de ello y manifestarlo sin pudor ni culpabilidad.

Una vez que los padres alcanzan la etapa de adaptación, comenzarán a preguntarse qué van a hacer en un futuro, y empieza a visualizarse el lazo afectivo hacia su hijo. Es ahí cuando debemos reforzar esta vinculación, mostrándoles señales de interacción que su hijo está logrando y educarlo sobre la vigilancia en la salud y sobre los programas de estimulación temprana, el rol de los padres de apoyo, así como la protección legal y social que ofrece el Estado.

Es por ello que el apego, especialmente en situaciones de discapacidad del bebé, se transforma en una emergencia afectiva, ya que repercutirá en el futuro del bebé. El equipo sanitario debe atender a las etapas por las que pasan los padres para detectar alguna anomalía (por ejemplo, duración prolongada o estancamiento en alguna de ellas) que pueda repercutir en el pequeño y la cual requiera la intervención de un profesional que le asista ante esa situación.

También es necesario estar alerta de las situaciones ambientales que contribuyan o favorezcan el deterioro de la vinculación afectiva, puesto que pueden entorpecer el desarrollo normal del niño. La desvinculación afectiva en el Síndrome de Down existe y es una realidad que debemos tener en cuenta. Por ello, es conveniente elaborar estrategias para evitarla o minimizarla en caso de que ya esté presente. Así, el equipo de salud debe informarse y establecer un plan de trabajo que consista en asistir emocionalmente a estas familias de la forma correcta y especializada.

\section{Referencias bibliográficas}

Ainsworth, M. D. S. (1983). Models of achievement: Reflections of prominent women in psychology. Nueva York:Columbia University Press.Atkinson, P., Coffey, A., \& Delamont, S. (1999). Ethnography: Post, Past and Present. Journal of Contemporary Ethnography, 28(5), 460-71.

Beeghly, M., Weiss-Perry, B., \& Cicchetti, D. (1990).

Beyond sensorimotor functioning: Early communicative and play development of children with Down syndrome. In D. Cicchetti \& M. Beeghly (Eds.), Children with Down syndrome: A developmental perspective (pp. 329-368). New York: Cambridge University Press.

Main, M., \& Solomon, J. (1990). Procedures for dentifying disorganized/disoriented infants during the Ainsworth Strange Situation. In M. Greenberg, D. Cicchetti \& M. Cummings (Eds), Attachment in the preschool years (pp. 121-160). Chicago: University of Chicago Press.

Rossel, K. (2004). Apego y vinculación en el Síndrome de Down. Una emergencia afectiva. Revista Pediatría Electrónica, 1(1), 3-8. Recuperado de: http://www.portalsindromededown.com.br/arquivos/ape go_e_down.pdf

Vaughn, B. E., \& Waters, E. (1990). Attachment behavior at home and in the laboratory: $Q$-sort observations and strange situation classifications of one-year-olds. Child Development, 61, 1965 - 1973.

Vaughn, B. E., Leftover, G. B., Seifer, R. \& Barglow, P. (1989). Attachment behavior, attachment and temperament during infancy. Child Development, 60,728-37.

Vaughn, B. E., Goldberg, S., Atkinson, L., Marcovitch, S., MacGregor, D., \& Seifer, R. (1994). Quality of toddler-mother attachment in children with Down syndrome: Limits to interpretation of strange situation behavior. Child Development, 65, 95-108. 